\title{
Spectrum of Normal and Pathologic Findings in the Region of the First Extensor Compartment of the Wrist
}

\author{
Sonographic Findings and Correlations \\ With Dissections
}

Michel De Maeseneer, MD, PhD, Stefaan Marcelis, MD, Tjeerd Jager, MD, Charles Girard, MD, Thomas Gest, PhD, David Jamadar, MD

\begin{abstract}
Objective. The purpose of this presentation is to review pathologic conditions that lead to pain at the radial aspect of the distal radius and to address anatomic variations of the first extensor compartment that exist and may have diagnostic and therapeutic implications. Methods. Our presentation is based on a review of cases from teaching files and observations made in anatomic specimens. Results. The discussed conditions include de Quervain tenosynovitis, intersection syndrome, and Wartenberg syndrome. Sonographic diagnosis of these conditions is addressed, and correlations are provided with anatomic specimens. Conclusions. Sonography is able to depict and differentiate between these conditions. Key words: de Quervain tenosynovitis; intersection syndrome; Wartenberg syndrome; wrist.
\end{abstract}

Abbreviations

MRI, magnetic resonance imaging

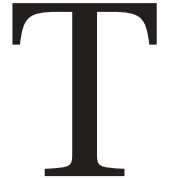

he first extensor compartment of the wrist is most typically involved in de Quervain tenosynovitis. Other ailments, however, exist, such as intersection syndrome and Wartenberg syndrome, which have to be differentiated from de Quervain tenosynovitis. Many anatomic variations of the first extensor compartment exist that may potentially lead to diagnostic errors and difficulty in diagnosis. Knowledge of the normal anatomy of the first extensor compartment is needed to avoid such errors. Magnetic resonance imaging (MRI) has typically been used for diagnosis of de Quervain tenosynovitis. In our practice, we have successfully used sonography for diagnosis and differentiation from other conditions. Our purpose in this presentation is to show the sonographic appearance of de Quervain tenosynovitis and to address anatomic variations and the differential diagnosis. 


\section{Materials and Methods}

The sonography teaching files of 3 institutions were reviewed by the principle investigator for representative cases. The studies were performed by 1 of 3 trained musculoskeletal radiologists. Five fresh anatomic specimens were used for anatomic observations. The specimens were obtained from 2 institutions. One specimen was sliced to show the relationships of the tendons at the wrist level. In 3 specimens, a dissection of the first extensor compartment was performed to study normal anatomy and variations. In 1 forearm, the course of the superficial branch of the radial nerve was studied. The anatomic material was reviewed by consensus of 2 musculoskeletal radiologists who selected the most representative material.

\section{Anatomic Considerations: Tendon Compartments}

There are 6 dorsal wrist compartments that respectively contain the abductor pollicis longus and extensor pollicis brevis, the extensor carpi radialis longus and brevis, the extensor pollicis longus, the extensor digitorum, the extensor digiti minimi, and the extensor carpi ulnaris (Figures 1 and 2). The compartments are covered by the extensor retinaculum (Figures 3 and 4). In the first compartment, the abductor pollicis longus has a larger diameter compared with the extensor pollicis brevis (Figures 5 and 6). The extensor carpi radialis tendons are located adjacent to the Lister tubercle. The Lister tubercle consists of a bony protrusion on the dorsal aspect of the radius toward the radial side of this bone. When the ultrasound transducer is placed in the transverse plane over the distal radius, the cortical protrusion can be easily identified (Figures 7 and 8).

\section{Anatomic Considerations: Intersection Area}

The tendons of the first compartment cross over the tendons from the second compartment about 3.5 to $4.8 \mathrm{~cm}$ proximal to the Lister tubercle (Figures 9 and 10). ${ }^{1}$ Muscle may already be evident at the level of the abductor pollicis longus representing the musculotendinous junction, and this should not be mistaken on sonography for peritendinous fluid (Figures 11 and 12). The hypoechoic appearance of the muscle is related to an anisotropy artifact and hence varies with transducer angulation. Synovial sheaths also may still be present at the crossover point.

\section{Variations of the First Compartment}

Several anatomic variations have been described in the literature, and some of these may have implications for diagnosis and therapy. The abductor pollicis longus is frequently multilamellar, and in a study by Kulthanan and Chareonwat, ${ }^{2}$ this was seen in $89 \%$ of specimens at dissection (Figures 13-15). This multilamellar appearance could be mistaken for a longitudinal

Figures 1 (top, drawing) and $\mathbf{2}$ (bottom, anatomic slice). The dorsal tendon compartments comprise the abductor pollicis longus and extensor pollicis brevis (1), extensor carpi radialis longus and brevis (2), extensor pollicis longus (3), extensor digitorum (4), extensor digiti minimi (5), and extensor carpi ulnaris (6) compartments
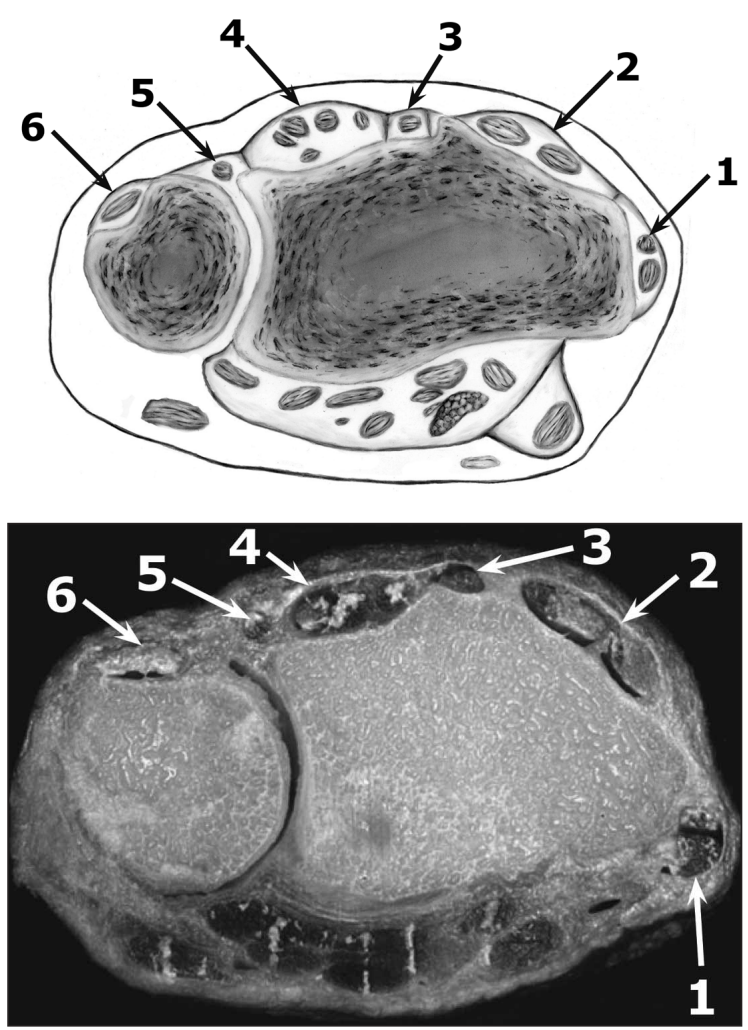


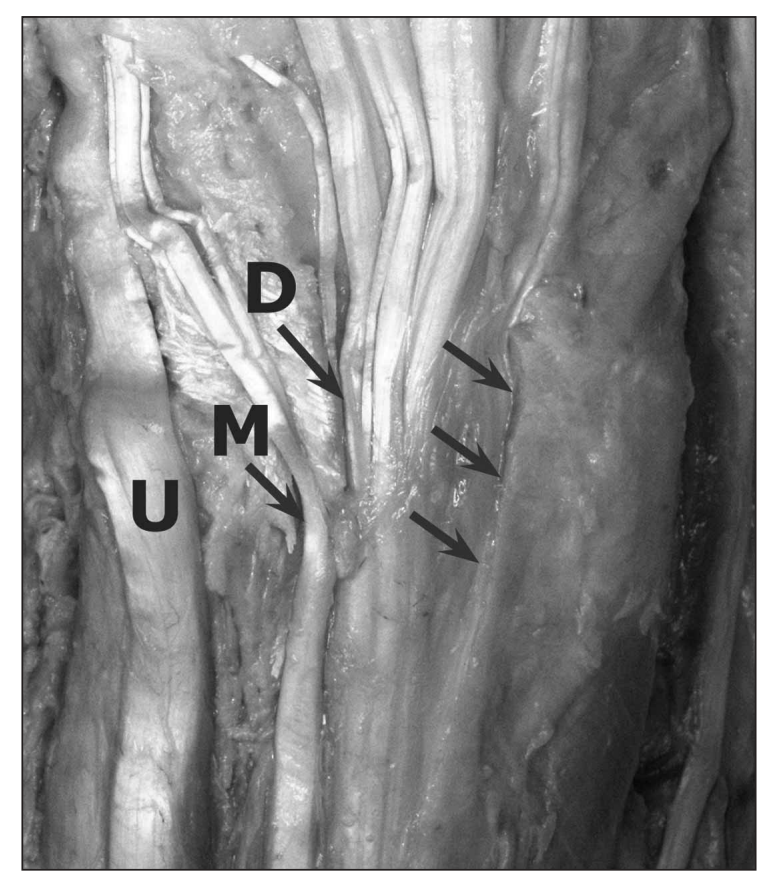

Figure 3. Anatomic dissection. The ulnar-sided compartments were opened, showing the extensor carpi ulnaris $(U)$, extensor digiti minimi (M), and extensor digitorum (D). Arrows indicate unopened radial-sided compartments with an intact retinaculum.

split tear, and care should be taken to avoid this diagnostic pitfall (Figure 16). ${ }^{3}$ Another common variation includes the presence of separate compartments for the abductor pollicis longus and extensor pollicis brevis (Figures 17 and 18). ${ }^{4}$ The septation appears somewhat similar to a tendon on sonography and could also lead to an erro-

Figure 4. Anatomic dissection. The radial-sided compartments were opened. Note the abductor pollicis longus (A), extensor pollicis brevis (arrow), extensor carpi radialis brevis (B) and longus $(\mathrm{L})$, and extensor pollicis longus $(\mathrm{P})$.

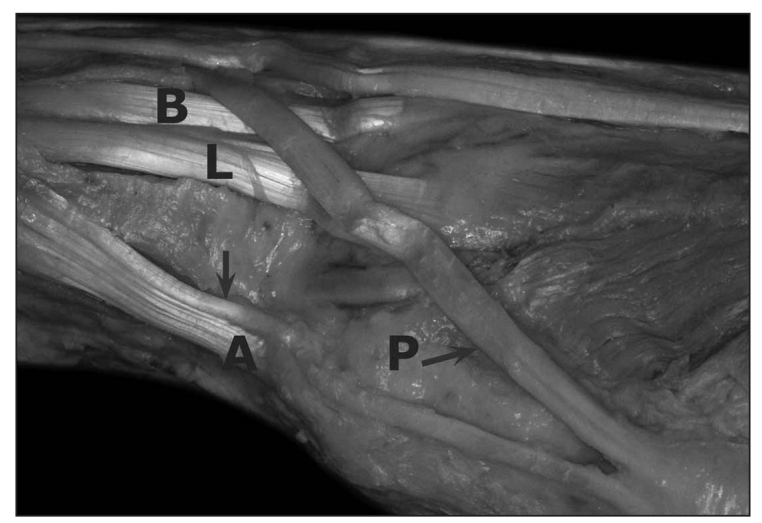

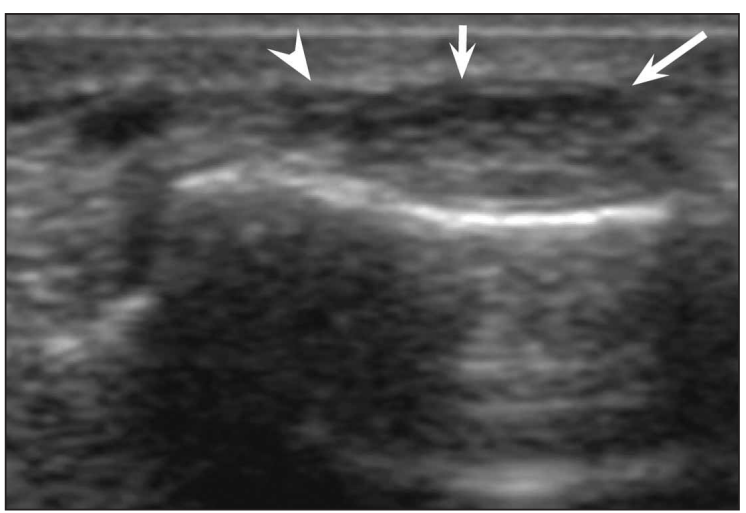

Figure 5. Sonogram from an asymptomatic 34-year-old woman. The first compartment is shown with the larger abductor pollicis longus (arrows) and smaller extensor pollicis brevis (arrowhead).

neous diagnosis of a longitudinal split tear (Figure 19). On a therapeutic level, the compartmentalization also may have implications. Infiltrations may be performed in only one of the compartments, leading to continuation of clinical symptoms. Furthermore, surgical decompression may only address one compartment, and this also may result in inadequate relief of symptoms.

\section{de Quervain Tenosynovitis}

First described by de Quervain, clinical symptoms of this entity include pain at the end of the distal radius radiating to the forearm or thumb, moderate swelling, sensitivity to pressure, and crepitation. ${ }^{5}$ Pathologic findings include thick-

Figure 6. Sonogram from a 27-year-old man showing a longitudinal view of the abductor pollicis longus (arrows). The retinaculum overlying both tendons of the first compartment is also shown (arrowheads).

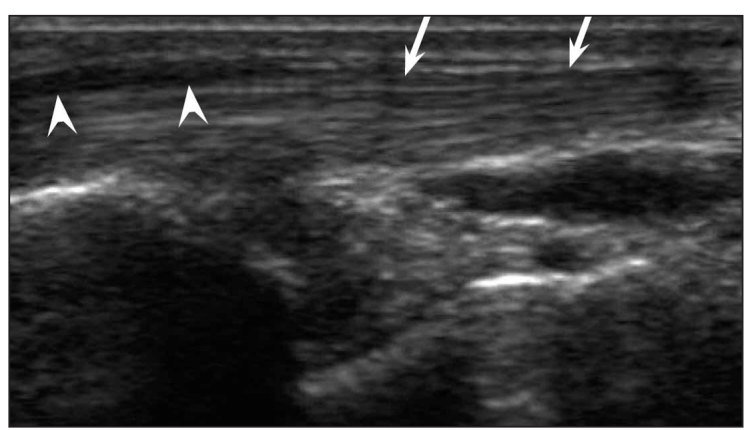




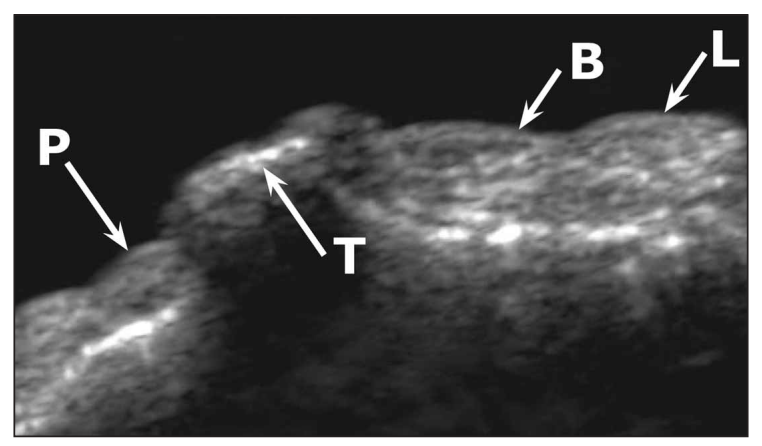

Figure 7. Sonogram from a cadaveric specimen. The extensor carpi radialis compartment with the extensor carpi radialis brevis (B) and longus ( $\mathrm{L}$ ) is shown. Also note the Lister tubercle ( $\mathrm{T}$ ) and extensor pollicis longus $(P)$.

ening of the retinaculum and tendovaginal chamber as well as the tendons. Radiographic findings typically are negative. On MRI, the thickening of the tendons may be seen, and the tendons may show an increased signal and be surrounded by a fluid collection in the tenosynovial sheath. ${ }^{6}$ On sonography, tendon thickening and tendon sheath effusion are apparent (Figures 20 and 21). The synovial sheath may be thickened in a lobulated fashion, and the retinaculum also may appear increased in size.,8 On Doppler sonography, marked hypervascularity may be evident (Figure 22). Clinical treatments include local infiltrations with nonsteroidal antiinflammatory drugs or steroidal medications and often surgical release of the compartment. ${ }^{5}$

Figure 8. Sonogram from an asymptomatic 40-year-old man. The normal extensor carpi radialis brevis $(B)$ and longus $(L)$ are shown.

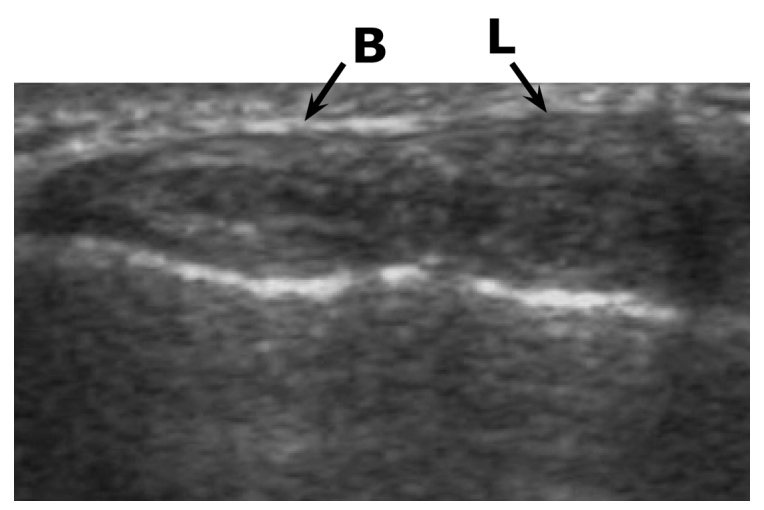

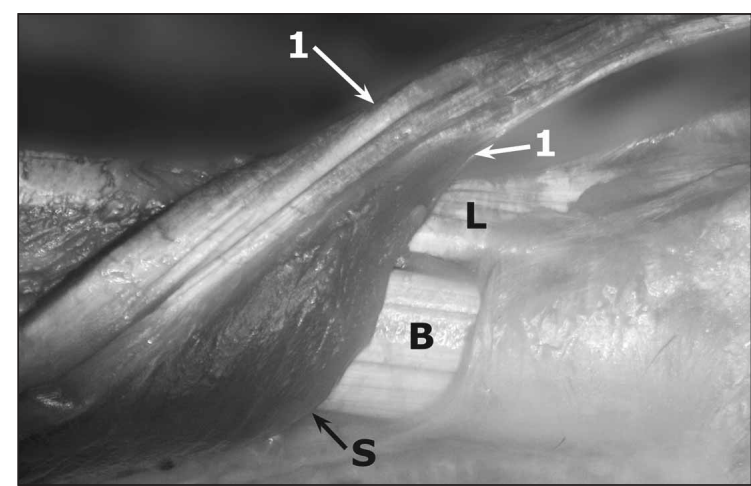

Figure 9. Anatomic dissection. The tendons of the first compartment cross over the tendons of the second compartment about 4 to $8 \mathrm{~cm}$ proximal to the Lister tubercle. In this region, a small muscle slip is commonly seen adjacent to the tendon ( $S$ ). Note the extensor carpi radialis brevis (B) and longus ( $\mathrm{L}$ ) as well as the first compartment tendons (1).

\section{Intersection Syndrome}

The precise cause of intersection syndrome still is not completely understood. A site of inflammatory changes typically occurs 4 to $8 \mathrm{~cm}$ proximal to the Lister tubercle at the intersection point between the first and second compartments. ${ }^{9}$ Localized pain is the main symptom, although swelling may also be palpated. It should be differentiated from the pain in de Quervain tenosynovitis, which is localized considerably more distally. The pathologic mechanism of intersection syndrome remains elusive. Radiographic findings, as in de Quervain tenosynovitis, are negative. On MRI, the tendons appear thickened, and fluid may be seen within the tendon sheaths.

Figure 10. Crossover point between the tendons of the first compartment (1) and extensor carpi radialis brevis (B) and longus (L). Note the muscle slip (S).

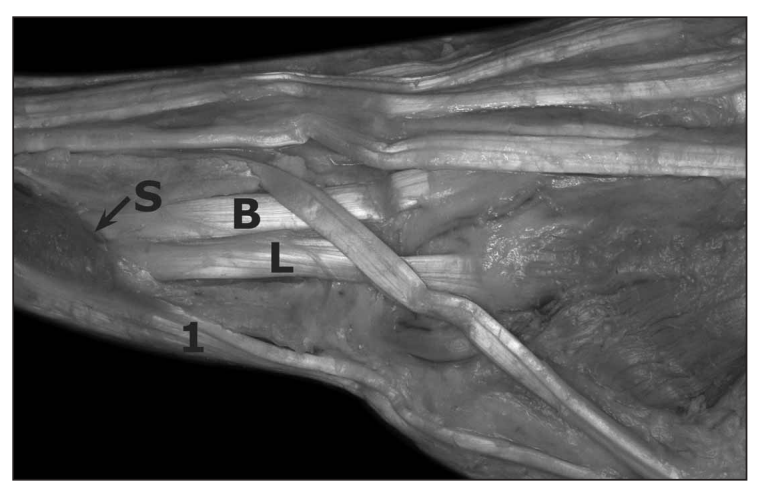




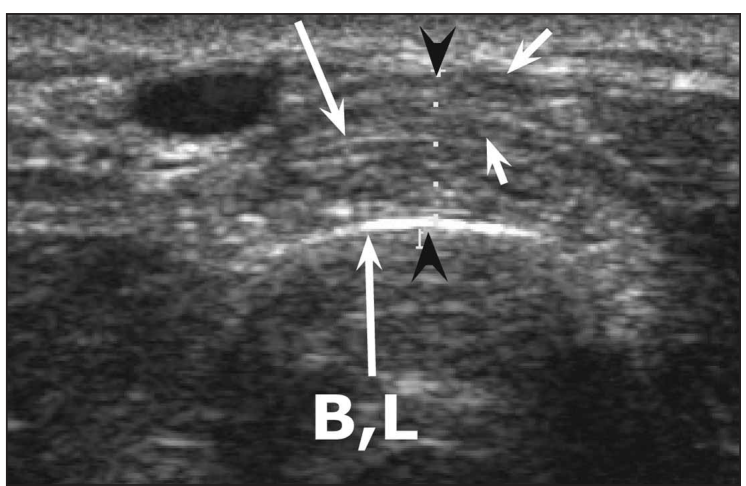

Figure 11. Normal sonogram from a 26-year-old man showing the intersection area. Note the extensor carpi radialis brevis (B) and longus $(\mathrm{L})$ between the long arrows and the first compartment tendons between the short arrows. Normal thickness at the intersection area is best appreciated in the transverse plane at the crossover point (arrowheads).

Peritendinous contrast enhancement also has been reported. ${ }^{1}$

Similar findings can be observed on sonography (Figures 23 and 24). The tendons appear markedly thickened, especially when compared with the contralateral asymptomatic side. Peritendinous fluid may be observed as well as peritendinous hypervascularity on Doppler sonography. Dynamic sonography could potentially be helpful to show friction between the tendons. Treatment consists of anti-inflammatory medications or infiltration with steroidal drugs. Surgical treatment also has been advocated by some authors. ${ }^{1,9}$ Modification of movement habits that sometimes provoke the disorders also can be considered.

Figure 12. Normal sonogram from a 56-year-old woman. An oblique transducer position shows the extensor carpi radialis brevis (B) and longus (L). Note the muscle slip (arrow) of the first compartment. This should not be mistaken for peritendinous fluid. The hypoechoic appearance is due to an anisotropy artifact and will change with the transducer position.

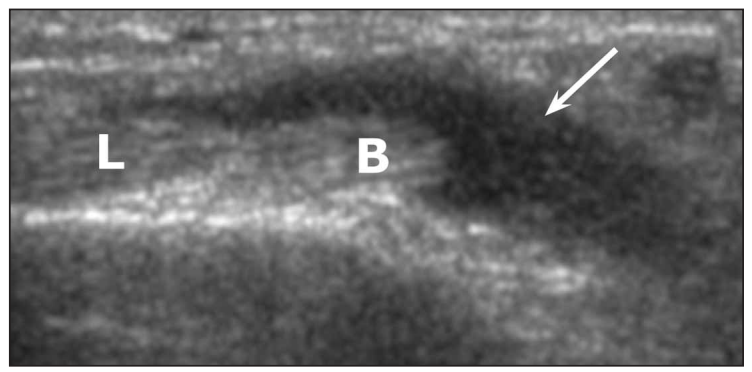

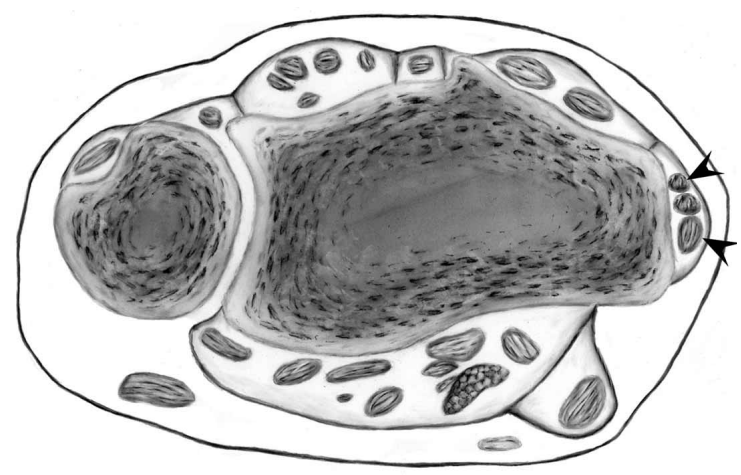

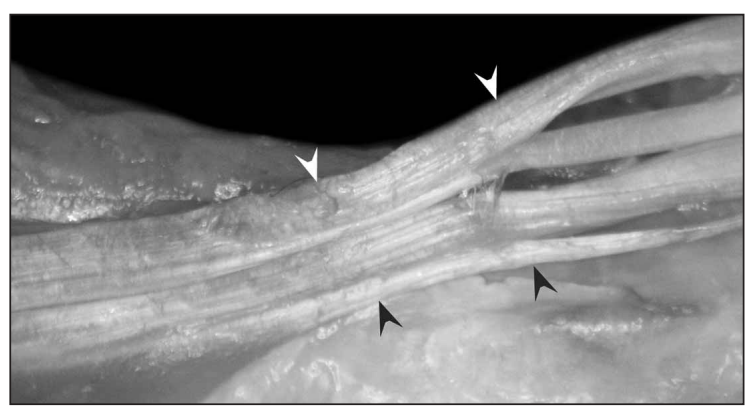

Figures $\mathbf{1 3}$ (top, drawing) and $\mathbf{1 4}$ (bottom, anatomic dissection) showing a multilamellar abductor pollicis longus (arrowheads). The multilamellar appearance should not be mistaken for longitudinal tears.

\section{Wartenberg Syndrome}

The superficial branch of the radial nerve becomes superficial at about $9 \mathrm{~cm}$ proximal to the styloid process of the radius (Figure 25). ${ }^{10}$ More distally, it divides into 3 separate branches. There appears a risk of nerve injury during placement of fixator pins due to Colles fractures, penetrating trauma, and cephalic vein cannulation. ${ }^{11}$

Figure 15. Anatomic dissection. Three tendons are shown at the distal first compartment due to a duplicated abductor pollicis longus, instead of the expected 2 tendons (arrowheads).

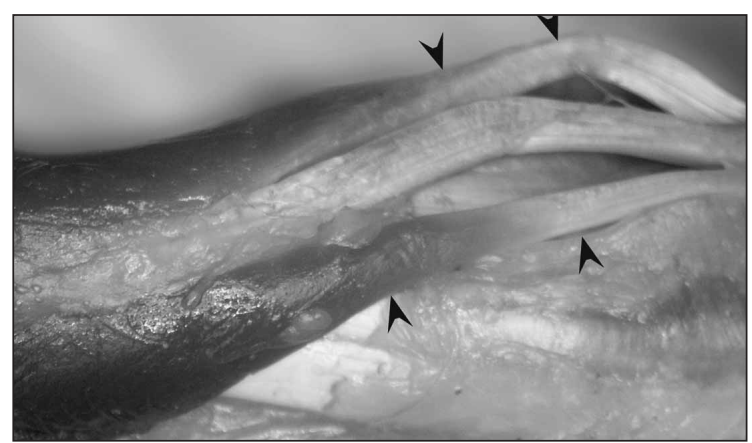




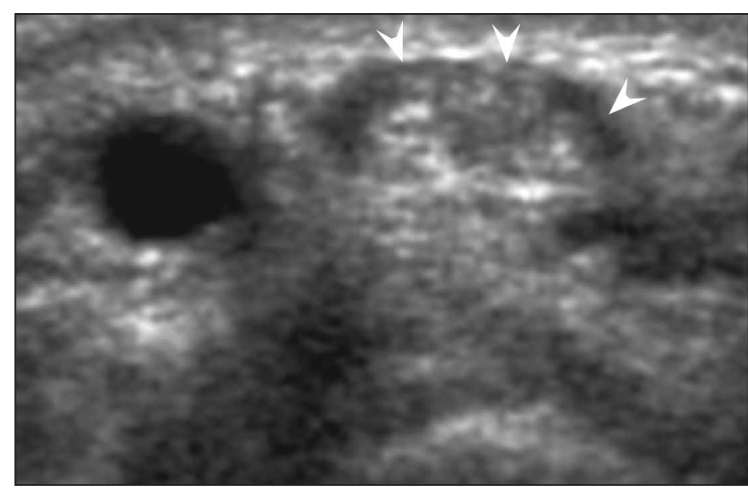

Figure 16. Sonogram from an asymptomatic 37-year-old man. Three separate tendons are shown (arrows). This should not be mistaken for evidence of a longitudinal split tear.

Wartenberg syndrome is not uncommonly associated with de Quervain tenosynovitis, although the reason for this association is not known. ${ }^{12,13}$ Clinical symptoms include pain, numbness, and paresthesias over the lateral aspect of the wrist and thumb, a somewhat similar territory to where symptoms of Quervain tenosynovitis manifest themselves. With electrodiagnostic tests, decreased sensory conduction velocity may be apparent. Radiographic findings tend to be negative, although in one case, a local bony spur emanating from the radius was described, which caused irritation of the nerve. ${ }^{13}$ Magnetic resonance imaging findings to our knowledge have not been reported. We speculate that the

Figure 17. Drawing of an additional septum. The abductor pollicis longus and extensor pollicis longus are located in separate compartments (arrows). This may lead to infiltrations that are performed in only one compartment or incomplete surgical release.

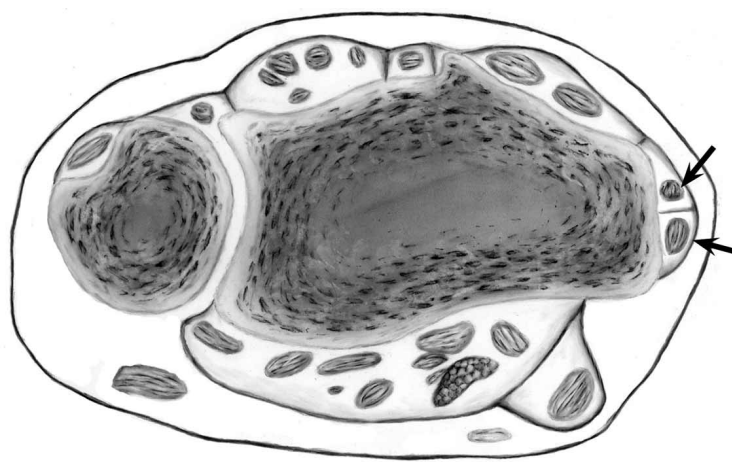

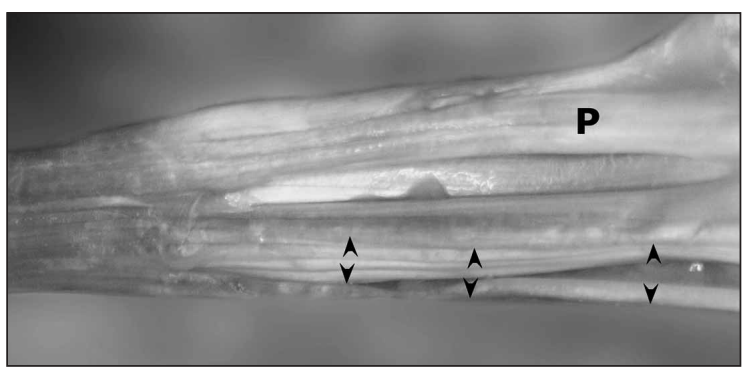

Figure 18. Anatomic dissection showing separate compartments. The compartment for the extensor pollicis brevis was opened (P). Note a partially opened separate compartment for the abductor pollicis longus (arrowheads).

nerve will be difficult to depict on MRI even with the use of dedicated coils. On sonography, the nerve can be well seen, and in the case of Wartenberg syndrome, an area of localized thickening is shown (Figure 26). Normal ranges for the thickness of this nerve have not been reported. However, we apply a comparison with the normal contralateral side to show any asymmetries. Therapy consists of surgical decompression.

\section{Osteoarthritis of the First Carpometacarpal Joint}

Osteoarthritis commonly affects the first carpometacarpal joint. Clinical findings may include swelling, tenderness, crepitus, and subluxation. Clinically, the condition may mimic de

Figure 19. Sonogram from a 62-year-old man with radial-sided pain and crepitus. Hypoechoic synovial effusion is shown (arrows). Three tendons are shown as well as a thinner area corresponding to the septum (S).

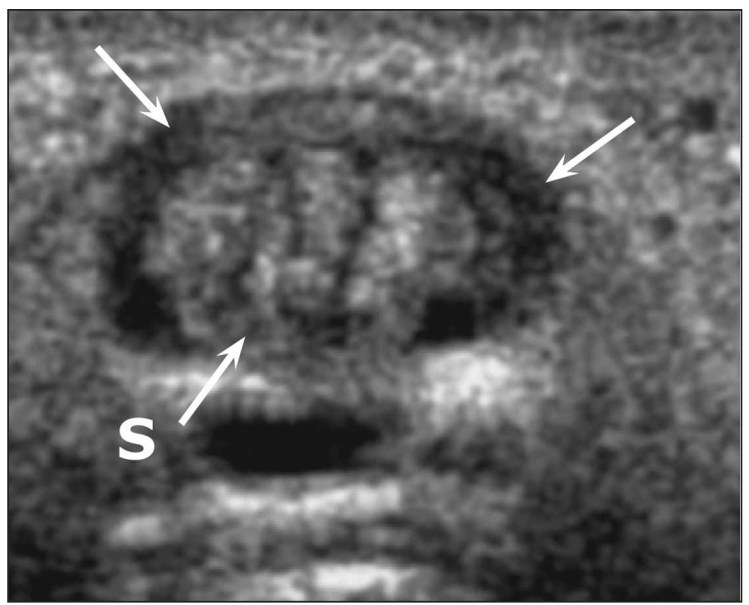



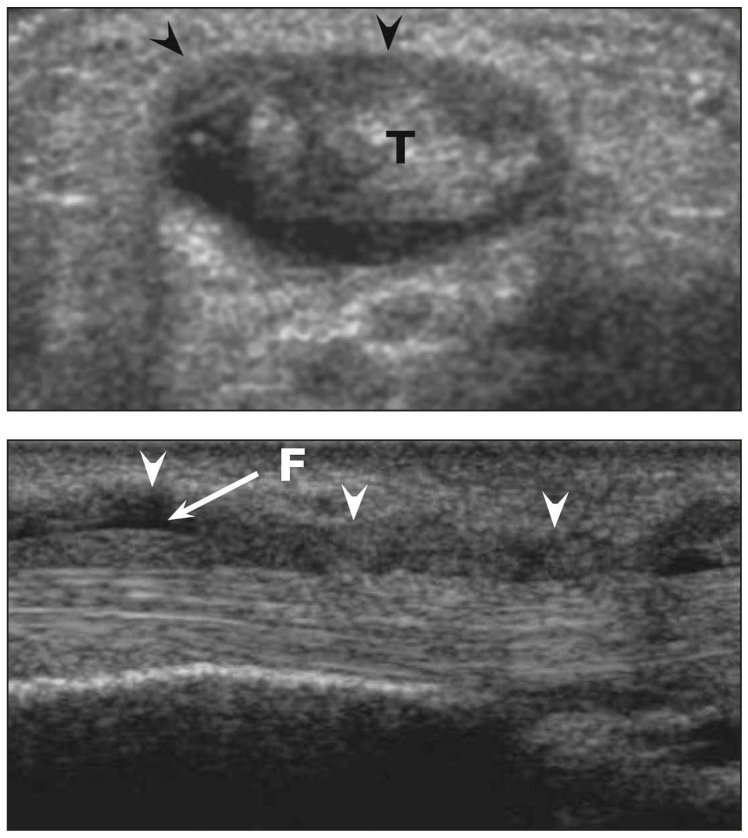

Figures $\mathbf{2 0}$ and $\mathbf{2 1}$. Sonograms from a 43-year-old woman with radial-sided pain. Typical sonographic findings are shown. In Figure 20 (top), tendon thickening ( $T$ ) and tendon sheath effusion (arrowheads) are shown. In Figure 21 (bottom), lobulated thickening of the synovial sheath and retinaculum is shown (arrowheads) as well as hypoechoic fluid (F).

Quervain tenosynovitis. Radiography shows a diminished joint space, osteophytes, subchondral sclerosis, and loose joint bodies. On sonography, similar findings are seen, and a joint effusion also may be apparent (Figure 27). Medical therapeutic options include splinting and infiltrations with nonsteroidal anti-inflammatory drugs or steroids. Surgical options include excision of the trapezium.

Figure 22. Sonogram from a 28-year-old man with radial-sided pain and crepitus. Marked hypervascularity is noted on Doppler imaging, in addition to synovial effusion (arrows). Note the separate compartments for the extensor pollicis brevis (B) and abductor pollicis longus ( $\mathrm{P}$.

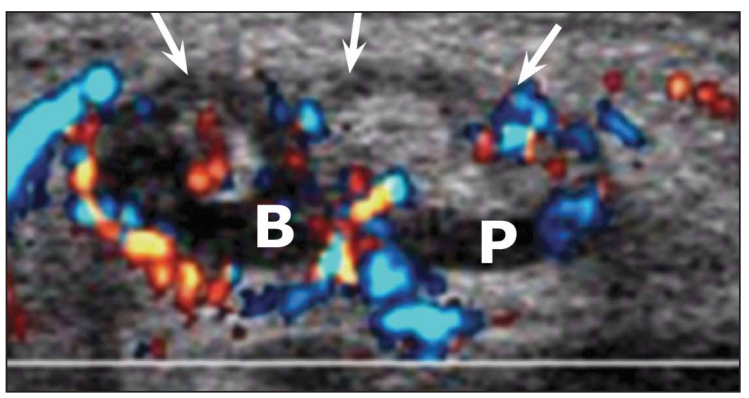

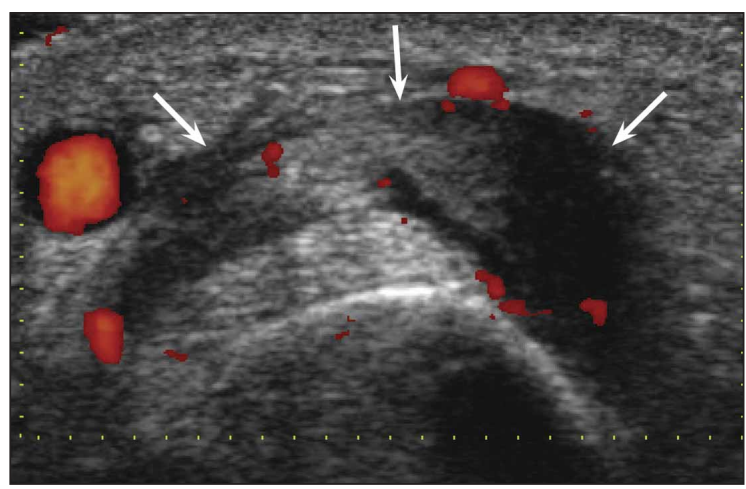

Figure 23. Sonogram from a 45-year-old woman. Clinical symptoms included pain, tenderness, and swelling. Note thickening of all of the tendons at the intersection, peritendinous fluid, and peritendinous hypervascularity on Doppler imaging (arrows).

\section{Conclusions}

We have reviewed the anatomy of structures at the distal radial aspect of the forearm and wrist and have described 3 conditions that may lead to similar symptoms and that should be differentiated from each other. Sonography is a valuable technique for diagnosing de Quervain tenosynovitis, intersection syndrome, and Wartenberg syndrome.

Figure 24. Sonogram from a 34-year-old man with pain and crepitus. Note marked thickening of the intersection area (left, arrowheads) compared with the normal side (right, arrowheads). A muscle slip of the first compartment (M) is shown.

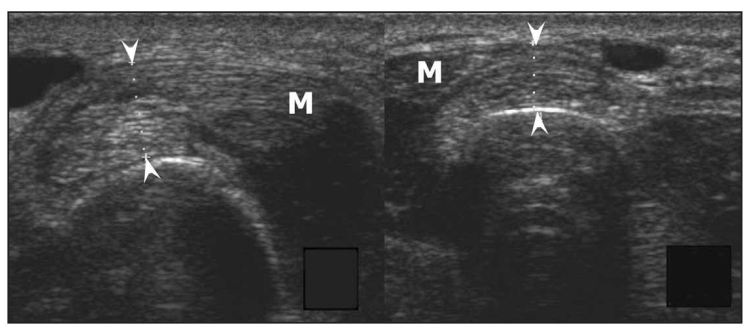



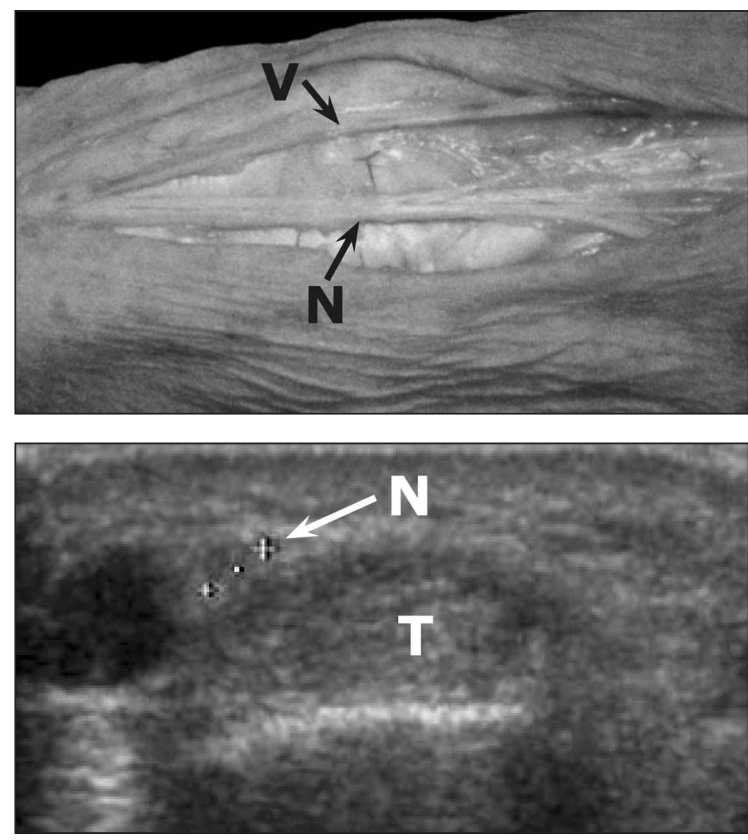

Figures $\mathbf{2 5}$ (top, anatomic dissection) and $\mathbf{2 6}$ (bottom, sonogram from a 32-year-old woman with pain and sensory changes at the lateral wrist). The proximity of the radial nerve $(\mathrm{N})$ branch to the venous $(V)$ structure is shown in Figure 25. A thickened nerve branch $(\mathrm{N})$ is shown in Figure 26 adjacent to the first compartment tendons $(\mathrm{T})$.

Figure 27. Sonogram of the first carpometacarpal joint from a 60 -year-old woman. Note loose bodies and joint effusion (arrows) at the first carpometacarpal joint.

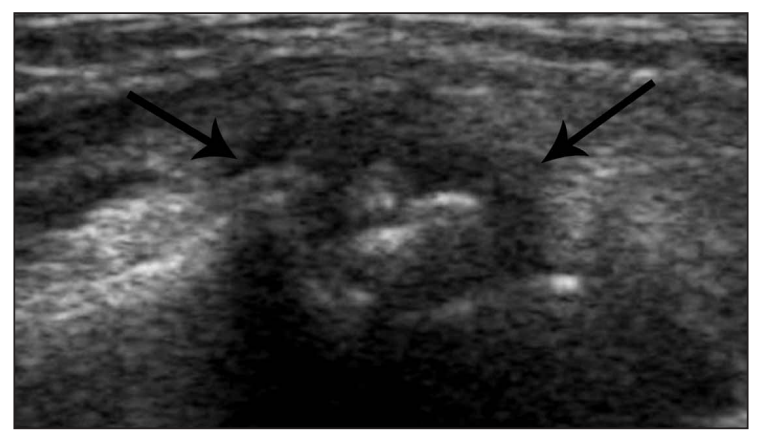

\section{References}

1. de Lima JE, Kim HJ, Albertotti F, Resnick D. Intersection syndrome: MR imaging with anatomic comparison of the distal forearm. Skeletal Radiol 2004; 33:627-631.

2. Kulthanan T, Chareonwat B. Variations in abductor pollicis longus and extensor pollicis brevis tendons in de Quervain syndrome: a surgical and anatomical study. Scand J Plast Reconstr Surg Hand Surg 2007; 41:36-38.

3. Shiraishi N, Matsumura G. Anatomical variations of the extensor pollicis brevis tendon and abductor pollicis longus tendon: relation to tenosynovectomy. Okijimas Folia Anat Jpn 2005; 82:25-29.

4. Hoch J, Fritch H, Lewejohann S. Congenital or acquired disposition of the separate compartment of the extensor pollicis brevis tendon associated with stenosing tendovaginitis (de Quervain disease)? Macro anatomical and fetal-plastination histological studies of the first compartment of the wrist [in German]. Ann Anat 2004; 186:305-310.

5. de Quervain F. On a form of chronic tendovaginitis by $\mathrm{Dr}$ Fritz de Quervain in la Chaux-de-Fonds, 1985. Am J Orthop 1997; 26:641-644.

6. Glajchen N, Schweitzer M. MRI features in de Quervain's tenosynovitis of the wrist. Skeletal Radiol 1996; 25:63-65.

7. Giovagnorio F, Andreoli C, De Cicco ML. Ultrasonographic evaluation of de Quervain disease. J Ultrasound Med 1997; 16:685-689.

8. Nagaoka M, Matsuzaki H, Suzuki T. Ultrasonographic examination of de Quervain's disease. J Orthop Sci 2000; 5:96-99.

9. Costa CR, Morrison WB, Carrino JA. MRI features of intersection syndrome of the forearm. AJR Am J Roentgenol 2003; 181:1245-1249.

10. Ikiz ZA, Ucerler $\mathrm{H}$. Anatomic characteristics and clinical importance of the superficial branch of the radial nerve. Surg Radiol Anat 2004; 26:453-458.

11. Braidwood AS. Superficial radial neuropathy. J Bone Joint Surg Br 1975; 57:380-383.

12. Lanzetta M, Foucher G. Entrapment of the superficial branch of the radial nerve (Wartenberg's syndrome): a report of 52 cases. Int Orthop 1993; 17:342-345.

13. Tosun N, Tuncay I, Akpinar F. Entrapment of the sensory branch of the radial nerve (Wartenberg's syndrome): an unusual cause. Tohoku J Exp Med 2001; 193:251-254. 